DEMONSTRATIO MATHEMATICA

Vol. XXVIII No $2 \quad 1995$

Magdalena Chrapek, Wiesław Dziubdziela

\title{
ON THE LIMIT DISTRIBUTIONS OF ORDER STATISTICS IN A SEQUENCE OF SOME 1-DEPENDENT RANDOM VARIABLES
}

\section{Introduction}

We present necessary and sufficient conditions for the weak convergence of the distributions of the $k$ th order statistics from some sequence of 1-dependent random variables. Limit laws are represented in terms of a compound Poisson distribution.

Let $\left\{\zeta_{n} ; n \geq 0\right\}$ be a sequence of i.i.d. random variables with values in a measurable space $(E, A)$ and with a common probability distribution $\pi$.

Let $\left\{\xi_{n}(x, y) ; n \geq 1\right\},(x, y) \in E \times E$, be a sequence of i.i.d. $A \times A-$ measurable random functions.

Assume that the sequences $\left\{\zeta_{n} ; n \geq 0\right\}$ and $\left\{\xi_{n}(x, y) ; n \geq 1\right\}$ are independent.

Consider the sequence of random variables:

$$
X_{1}=\xi_{1}\left(\zeta_{0}, \zeta_{1}\right), X_{2}=\xi_{2}\left(\zeta_{1}, \zeta_{2}\right), \ldots, X_{n}=\xi_{n}\left(\zeta_{n-1}, \zeta_{n}\right), \ldots
$$

Denote by

$$
\min \left(X_{1}, \ldots, X_{n}\right)=M_{n}^{(n)} \leq M_{n}^{(n-1)} \leq \ldots \leq M_{n}^{(1)}=\max \left(X_{1}, \ldots, X_{n}\right)
$$

the order statistics of the sequence $X_{1}, \ldots, X_{n}$.

M. O. Djnaid in [1] has obtained limit laws for $M_{n}^{(1)}$. In this paper we shall be concerned with conditions under which, for suitable normalizing constants $\left\{a_{n}>0, b_{n} ; n \geq 1\right\}$,

Key words and phrases: weak convergence, 1-dependent random variables, compound Poisson distributions. 


$$
P\left\{M_{n}^{(k)} \leq a_{n} x+b_{n}\right\} \stackrel{w}{\rightarrow} G^{(k)}(x), \quad k=1,2, \ldots,
$$

where $G^{(k)}$ is the distribution function and the notation $\stackrel{w}{\rightarrow}$ denotes the weak convergence of the distribution functions. We shall also be interested in forms of limit distribution $G^{(k)}$.

\section{Main results}

While obtaining the necessary and sufficient conditions for convergence (2) we use the results from the paper by W. N. Hudson et al. [3].

Let $I_{n j}(x)=I_{\left\{X_{j}>a_{n} x+b_{n}\right\}}, n=1,2, \ldots, j=1,2, \ldots, n$, where $I_{A}$ denotes the indicator function of the set $A$ and let

$$
J_{n}(x)=\sum_{j=1}^{n} I_{n j}(x), \quad n=1,2, \ldots
$$

It is well known that, for $k=1,2, \ldots$,

$$
P\left\{M_{n}^{(k)} \leq a_{n} x+b_{n}\right\}=P\left\{J_{n}(x)<k\right\}, \quad-\infty<x<\infty .
$$

Now, we shall prove the following results:

THEOREM 1. The convergence (2) holds if and only if

$$
n \int_{E} \int_{E}\left(1-F\left(x_{0}, x_{1} ; a_{n} x+b_{n}\right)\right) \pi\left(d x_{0}\right) \pi\left(d x_{1}\right) \text { converges }
$$

and

$$
\begin{gathered}
n \iint_{E} \int_{E} \int_{E} F\left(x_{0}, x_{1} ; a_{n} x+b_{n}\right)\left(1-F\left(x_{1}, x_{2} ; a_{n} x+b_{n}\right)\right) . \\
\cdot F\left(x_{2}, x_{3} ; a_{n} x+b_{n}\right) \pi\left(d x_{0}\right) \pi\left(d x_{1}\right) \pi\left(d x_{2}\right) \pi\left(d x_{3}\right) \rightarrow \lambda_{1}(x), \\
n \iiint_{E} \int_{E} F\left(x_{0}, x_{1} ; a_{n} x+b_{n}\right)\left(1-F\left(x_{1}, x_{2} ; a_{n} x+b_{n}\right)\right) . \\
\cdot\left(1-F\left(x_{2}, x_{3} ; a_{n} x+b_{n}\right)\right) \pi\left(d x_{0}\right) \pi\left(d x_{1}\right) \pi\left(d x_{2}\right) \pi\left(d x_{3}\right) \rightarrow \lambda_{2}(x),
\end{gathered}
$$

where $F(x, y ; u)=P\left\{\xi_{n}(x, y) \leq u\right\}$.

The limit distribution functions $G^{(k)}(x)$ are given by the formula:

$$
G^{(k)}(x)=\sum_{s=0}^{k-1} P\left\{s, \lambda_{1}(x), \lambda_{2}(x)\right\},
$$


for $x$ such that $0<G^{(k)}(x)<1$, where

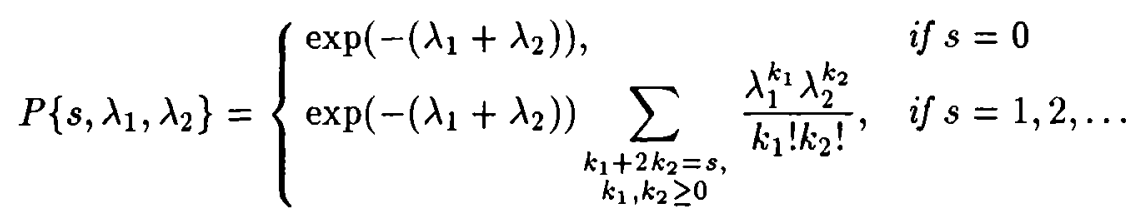

( Here we take $0^{0}=1$ ).

P r o of. It is clear that the sequence $\left\{X_{n} ; n \geq 1\right\}$ defined by (1) is strictly stationary and 1-dependent. Hence, the random variables $\left\{I_{n j}(x) ; n \geq 1\right\}$ are strictly stationary and 1-dependent in each row. It follows from Theorem 2 in [3] that the sequence $\left\{J_{n}(x) ; n \geq 1\right\}$ weakly converges (as $n \rightarrow \infty$ ) if and only if

$$
n P\left\{I_{n 1}=1\right\}=n \iint_{E}\left(1-F\left(x_{0}, x_{1} ; a_{n} x+b_{n}\right)\right) \pi\left(d x_{0}\right) \pi\left(d x_{1}\right)
$$

converges and the conditions (4) and (5) hold. Moreover, the limit distribution of $I_{n}(x)$ has a characteristic function given by the formula

$$
\phi(t)=\exp \left(\sum_{n=1}^{2} \lambda_{n}(x)\left(e^{i \ell n}-1\right)\right) .
$$

Note that it is the characteristic function of the compound Poisson distribution (see eg. [2]). Using (3) we complete the proof.

Now, we give two examples illustrating the main result.

EXAMPLE 1. Let $\left\{\zeta_{n} ; n \geq 0\right\}$ be a sequence of i.i.d. random variables with the exponential distribution with the parameter 1 . Let $\xi_{n}(x, y)=\max (x, y)$, $x, y \in R, n \geq 1$.

It is easy to compute that for $a_{n}=1, b_{n}=\ln (n), n=1,2, \ldots$, we have $\lambda_{1}(x)=0, \lambda_{2}(x)=e^{-x}$.

EXAMPLE 2. Let $\left\{\zeta_{n} ; n \geq 0\right\}$ and $\left\{a_{n}, b_{n} ; n \geq 1\right\}$ be the same as in Example 1. Let $\left\{N_{n} ; n \geq 1\right\}$ be a sequence of i.i.d. Bernoulli random variables with a parameter $a=P\left\{N_{1}=1\right\}=1-P\left\{N_{1}=0\right\}, 0<a<1$. Assume that $\left\{\zeta_{n} ; n \geq 0\right\}$ and $\left\{N_{n} ; n \geq 1\right\}$ are independent.

Define

$$
X_{n}= \begin{cases}\zeta_{n-1}, & \text { if } N_{n}=1 \\ \zeta_{n}, & \text { if } N_{n}=0 .\end{cases}
$$

By Theorem 1 we obtain $\lambda_{1}(x)=2 e^{-x}\left(2 a^{2}-2 a+1\right), \lambda_{2}(x)=2 e^{-x}\left(a-a^{2}\right)$.

As an easy consequence of Theorem 1 we have the following corollary:

Corollary 1. Let $\left\{X_{n} ; n \geq 1\right\}$ defined by (1) satisfy the following assumption: 
Assumption A. There exist normalizing constants $\left\{a_{n}>0, b_{n} ; n \geq 1\right\}$ and a nondegenerate distribution function $G$ such that

$$
\begin{aligned}
\lim _{n \rightarrow \infty} n \iint_{E}\left(1-F\left(x_{0}, x_{1} ; a_{n} x+b_{n}\right)\right) & \pi\left(d x_{0}\right) \pi\left(d x_{1}\right)= \\
& = \begin{cases}\infty, & \text { if } G(x)=0 \\
-\log G(x), & \text { if } G(x)>0 .\end{cases}
\end{aligned}
$$

Moreover, assume that

$$
\lim _{n \rightarrow \infty} \sup _{x}\left(1-\int_{E} F\left(x, y ; a_{n} x+b_{n}\right) \pi(d y)\right)=0 .
$$

Then,

$$
P\left\{M_{n}^{(k)} \leq a_{n} x+b_{n}\right\} \stackrel{w}{\rightarrow} \begin{cases}0, & \text { if } G(x)=0 \\ \sum_{s=0}^{k-1} \frac{1}{s !}(-\log G(x))^{s}, & \text { if } 0<G(x)<1 \\ 1, & \text { if } G(x)=1,\end{cases}
$$

for $k=1,2, \ldots$.

Proof. By Theorem 1 it is sufficient to show that $\lambda_{1}(x)=-\log G(x)$, $\lambda_{2}(x)=0$, where $\lambda_{1}(x)$ and $\lambda_{2}(x)$ are given by the formulas (4) and (5). By stationarity we have

$\iiint \int F\left(x_{0}, x_{1} ; a_{n} x+b_{n}\right)\left(1-F\left(x_{1}, x_{2} ; a_{n} x+b_{n}\right)\right)\left(1-F\left(x_{2}, x_{3} ; a_{n} x+b_{n}\right)\right)$. EEEE

$$
\begin{aligned}
& \cdot \pi\left(d x_{0}\right) \pi\left(d x_{1}\right) \pi\left(d x_{2}\right) \pi\left(d x_{3}\right) \leq \\
& \leq \iint_{E} \int_{E}\left(1-F\left(x_{0}, x_{1} ; a_{n} x+b_{n}\right)\right)\left(1-F\left(x_{1}, x_{2} ; a_{n} x+b_{n}\right)\right) \pi\left(d x_{0}\right) \pi\left(d x_{1}\right) \pi\left(d x_{2}\right) \text {. }
\end{aligned}
$$

Since

$$
\begin{aligned}
& \int_{E} \int_{E}\left[\left(1-F\left(x_{0}, x_{1} ; a_{n} x+b_{n}\right)\right) \cdot\right. \\
& \left.\cdot\left(1-\int_{E} F\left(x_{1}, x_{2} ; a_{n} x+b_{n}\right) \pi\left(d x_{2}\right)\right)\right] \pi\left(d x_{0}\right) \pi\left(d x_{1}\right) \leq \\
& \leq\left(\iint_{E} \pi\left(d x_{0}\right) \pi\left(d x_{1}\right)-\iint_{E} F\left(x_{0}, x_{1} ; a_{n} x+b_{n}\right) \pi\left(d x_{0}\right) \pi\left(d x_{1}\right)\right) . \\
& \cdot \sup _{x_{1}}\left(1-\int_{E} F\left(x_{1}, x_{2} ; a_{n} x+b_{n}\right) \pi\left(d x_{2}\right)\right)
\end{aligned}
$$

so, by the assumptions of Corollary 1 , we obtain that $\lambda_{2}(x)=0$. 
Furthermore, we have:

$$
\begin{array}{r}
n \iint_{E} \int_{E} \int_{E} F\left(x_{0}, x_{1} ; a_{n} x+b_{n}\right)\left(1-F\left(x_{1}, x_{2} ; a_{n} x+b_{n}\right)\right) \cdot F\left(x_{2}, x_{3} ; a_{n} x+b_{n}\right) \\
\cdot \pi\left(d x_{0}\right) \pi\left(d x_{1}\right) \pi\left(d x_{2}\right) \pi\left(d x_{3}\right)= \\
=n \int_{E} \int_{E}\left(1-F\left(x_{0}, x_{1} ; a_{n} x+b_{n}\right)\right) \pi\left(d x_{0}\right) \pi\left(d x_{1}\right)- \\
-n \iint_{E} \int_{E}\left(1-F\left(x_{0}, x_{1} ; a_{n} x+b_{n}\right)\right)\left(1-F\left(x_{1}, x_{2} ; a_{n} x+b_{n}\right)\right) \pi\left(d x_{0}\right) \pi\left(d x_{1}\right) \pi\left(d x_{2}\right)- \\
-n \iint_{E} \int_{E} F\left(x_{0}, x_{1} ; a_{n} x+b_{n}\right)\left(1-F\left(x_{1}, x_{2} ; a_{n} x+b_{n}\right)\right) . \\
\cdot\left(1-F\left(x_{2}, x_{3} ; a_{n} x+b_{n}\right)\right) \pi\left(d x_{0}\right) \pi\left(d x_{1}\right) \pi\left(d x_{2}\right) \pi\left(d x_{3}\right) .
\end{array}
$$

Thus, by the assumptions, the minuend tends to $-\log G(x)$ and the subtrahends tend to 0 , so that $\lambda_{1}(x)=-\log G(x)$.

Remark 1. For $k=1$ from Corollary 1 we obtain the result of [1].

Remark 2. There is another way to obtain some results which are contained in Theorem 1. Namely, the following theorem may be obtained as a consequence of Theorem 4 of [2]. let

Theorem 2. Let $\left\{X_{n} ; n \geq 1\right\}$ defined by (1) satisfy Assumption $A$ and

$$
G^{(k)}(x)= \begin{cases}0, & \text { if } G(x)=0 \\ \sum_{s=0}^{k-1} H(s ; G(x), d), & \text { if } 0<G(x)<1 \\ 1, & \text { if } G(x)=1, k=1,2, \ldots,\end{cases}
$$

where

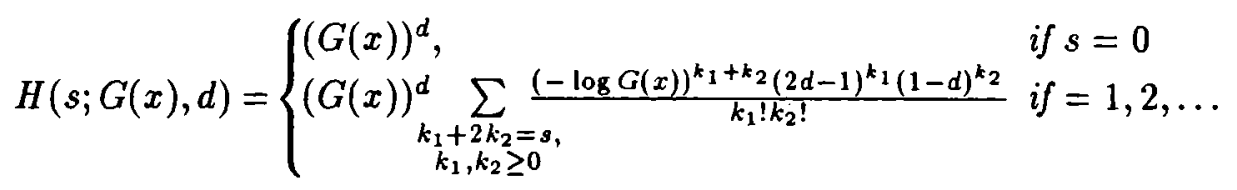

and $0.5 \leq d \leq 1$.

The convergence (2) holds if and only if

$$
\begin{aligned}
& \lim _{n \rightarrow \infty} n \iint_{E} \int_{E}\left(1-F\left(x_{0}, x_{1} ; a_{n} x+b_{n}\right)\right) . \\
& \quad \cdot\left(1-F\left(x_{1}, x_{2} ; a_{n} x+b_{n}\right)\right) \pi\left(d x_{0}\right) \pi\left(d x_{1}\right) \pi\left(d x_{2}\right)=-(1-d) \log G(x),
\end{aligned}
$$

for all $x$ such that $0<G(x)<1$. 


\section{References}

[1] М. О. Джнейд, О предельном распределемии максимума некоторых случаймых өеличия, Theor. Verojatnost. i Mat. Statist. 37 (1987), 36-39.

[2] W. Dziubdziela, Limit laws for kth order statistics from strong-mixing processes, J. Appl. Probability 21 (1984), 720-729.

[3] W. N. Hudson, H. G. Tucker, J. A. Veeh, Limit distributions of sums of mdependent Bernoulli variables, Probab. Th. Rel. Fields 82 (1989), 9-17.

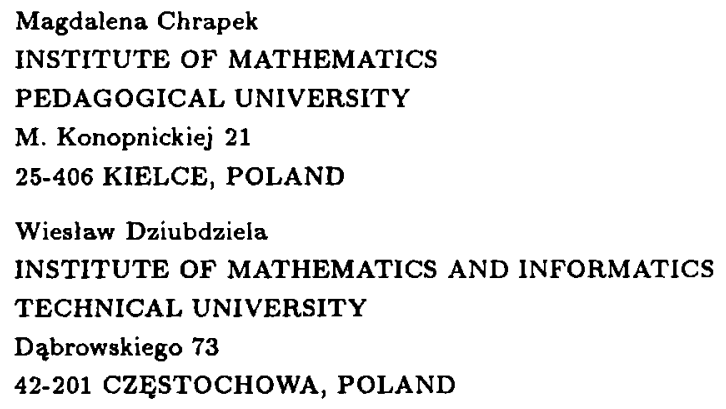

Received April 6, 1993. 